\title{
Shell Model Monte Carlo Method for Two-Neutrino Double Beta Decay
}

\author{
P. B. Radha, ${ }^{1}$ D. J. Dean, ${ }^{1,2}$ S. E. Koonin, ${ }^{1}$ T. T. S. Kuo, ${ }^{3}$ K. Langanke, ${ }^{1}$ A. Poves,${ }^{1,4}$ J. Retamosa,${ }^{4}$ and P. Vogel ${ }^{5}$ \\ ${ }^{1}$ W. K. Kellogg Radiation Laboratory, California Institute of Technology, Pasadena, California 91125 \\ ${ }^{2}$ Physics Division, Oak Ridge National Laboratory, P.O. Box 2008, Oak Ridge, Tennessee 37381 \\ ${ }^{3}$ Physics Department, SUNY at Stony Brook, Stony Brook, New York 11794 \\ ${ }^{4}$ Departmento de Física Teórica C-XI, Universidad Atuónoma de Madrid, E-28049 Madrid, Spain \\ ${ }^{5}$ Division of Physics, Mathematics and Astronomy, California Institute of Technology, Pasadena, California 91125
}

(Received 24 October 1995)

\begin{abstract}
Shell model Monte Carlo techniques are used to calculate two-neutrino double beta decay matrix elements. We test the approach against direct diagonalization for ${ }^{48} \mathrm{Ca}$ in the complete $p f$ shell using the KB3 interaction. The method is then applied to the decay of ${ }^{76} \mathrm{Ge}$ in the $\left(0 f_{5 / 2}, 1 p, 0 g_{9 / 2}\right)$ model space using a newly calculated realistic interaction. Our result for the matrix element is $0.12 \pm 0.05 \mathrm{MeV}^{-1}$, in reasonable agreement with the experimental value.
\end{abstract}

PACS numbers: 21.60.Cs, 21.60.Ka, 27.40.+z, 23.40.-s

The double beta $(\beta \beta)$ decay of a nucleus is a rare second order weak process $[1,2]$. The as-yet unobserved neutrinoless mode is of fundamental interest, as it would signal a neutrino mass, lepton number nonconservation, or admixtures of right handed weak currents. In contrast, the existence of the $2 \nu$ mode has been firmly established (see the review in Ref. [2]). The ability to accurately describe this latter process is an important element in the interpretation of limits on neutrinoless decays. Unfortunately, it seems that $2 \nu$ matrix elements are highly suppressed and so depend sensitively on small, poorly determined parts of the nuclear wave functions.

Most recent calculations of $2 \nu \beta \beta$ matrix elements for nuclei heavier than ${ }^{48} \mathrm{Ca}$ rely on the quasiparticle random phase approximation (QRPA) [2]. While this approach is computationally simple and includes many features of the two-body interaction known to be relevant for $\beta \beta$ decay, the calculated matrix elements are uncertain because of their great sensitivity to the $J=1^{+}, T=0$ particleparticle interaction [3]. The interacting shell model offers a more microscopic approach to the problem. Complete $0 \hbar \omega$ shell model calculations [4] not only recover more quenching of Gamow-Teller (GT) transitions than QRPA calculations, but also are in agreement with observations (after the universal renormalization of $g_{A}$ to 1.0). However, computational limitations have restricted shell model calculations of the $2 \nu \beta \beta$ decay matrix element to ${ }^{48} \mathrm{Ca}$, the lightest of all $\beta \beta$ candidates.

In this Letter we show how shell model Monte Carlo (SMMC) methods can be used to calculate $2 \nu \beta \beta$ decay matrix elements. We first calculate the decay of ${ }^{48} \mathrm{Ca}$ in the complete $p f$ shell and validate our method against direct diagonalization. We then present results for the decay of ${ }^{76} \mathrm{Ge}$, one of the few nuclei where the $2 \nu \beta \beta$ decay has been precisely measured and where the best limits on the $0 \nu$ decay have been obtained [5]. Our calculation in the complete $\left(0 f_{5 / 2}, 1 p, 0 g_{9 / 2}\right)$ orbitals, which is impractical using traditional shell model methods, is the first of $2 \nu \beta \beta$ decay in such a large model space.
The $2 \nu \beta \beta$ matrix elements between the $0^{+}$ground states of the initial and final even-even nuclei is given by [6]

$$
M^{2 \nu}=\sum_{m} \frac{\left\langle 0_{f}^{+}|\mathbf{G}| 1_{m}^{+}\right\rangle \cdot\left\langle 1_{m}^{+}|\mathbf{G}| 0_{i}^{+}\right\rangle}{E_{m}-\left(E_{i}^{0}+E_{f}^{0}\right) / 2} .
$$

Here, $\left|0_{i}^{+}\right\rangle\left(\left|0_{f}^{+}\right\rangle\right)$is the ground state of the initial (final) nucleus with energy $E_{i}^{0}\left(E_{f}^{0}\right) ;\left|1_{m}^{+}\right\rangle$are the $1^{+}$states of the intermediate odd-odd nucleus with energies $E_{m}$; and $\mathbf{G}$ is the GT operator $\sum_{l} \boldsymbol{\sigma}_{l} \tau_{l}^{-}$, where $\boldsymbol{\sigma}_{l}$ is the Pauli spin operator for nucleon $l$ and $\tau_{l}^{-}$is the isospin lowering operator that changes a neutron into a proton.

Previous shell model calculations for nuclei heavier than ${ }^{48} \mathrm{Ca}$ ([1], and references therein) have invoked the so-called closure approximation, where the matrix element is written as $M^{2 \nu}=M_{c} / \tilde{E}$, with $M_{c}=\left\langle 0_{f}^{+}\right| \mathbf{G}$. $\mathbf{G}\left|0_{i}^{+}\right\rangle$and $\bar{E}$ an average energy denominator. As there is no prescription for choosing the average energy denominator (and even the closure matrix element is usually calculated in a severely truncated basis), the uncertainty in this approximation is difficult to estimate.

To calculate the exact $2 \nu \beta \beta$ matrix element, Eq. (1), we consider the function

$$
\phi\left(\tau, \tau^{\prime}\right)=\frac{\operatorname{Tr}\left[e^{-\left(\beta-\tau-\tau^{\prime}\right) H} \mathbf{G}^{\dagger} \cdot \mathbf{G}^{\dagger} e^{-\tau H} \mathbf{G} e^{-\tau^{\prime} H} \cdot \mathbf{G}\right]}{\operatorname{Tr}\left[e^{-\beta H}\right]},
$$

where $H$ is the many-body Hamiltonian and the trace is over all states of the initial nucleus. The quantities $\beta-$ $\tau-\tau^{\prime}$ and $\tau$ play the role of the inverse temperature in the parent and daughter nucleus, respectively. A spectral expansion of $\phi$ shows that large values of these parameters guarantee cooling to the parent and daughter ground states. In these limits, we note that $\phi\left(\tau, \tau^{\prime}=\right.$ $0)$ approaches $e^{-\tau Q}\left|M_{c}\right|^{2}$, where $Q=E_{i}^{0}-E_{f}^{0}$ is the energy release, so that a calculation of $\phi(\tau, 0)$ leads 
directly to the closure matrix element. If we then define

$$
\eta(T, \tau) \equiv \int_{0}^{T} d \tau^{\prime} \phi\left(\tau, \tau^{\prime}\right) e^{-\tau^{\prime} Q / 2}
$$

and

$$
M^{2 \nu}(T, \tau) \equiv \frac{\eta(T, \tau) M_{c}^{*}}{\phi(\tau, 0)},
$$

it is easy to see that in the limit of large $\tau, \beta-$ $\tau-\tau^{\prime}$, and $T, M^{2 \nu}(T, \tau)$ becomes independent of these parameters and is equal to the matrix element in Eq. (1).

We use SMMC methods [7] to calculate $\phi\left(\tau, \tau^{\prime}\right)$, and hence $M^{2 \nu}$. These techniques scale more gently than direct diagonalization with the number of valence nucleons and single particle orbits and so allow calculations larger then possible otherwise. They are based on the discretization of the many-body propagator $e^{-\beta H}$ into a finite number of "time" slices $N_{t}$, each of duration $\Delta \beta=\beta / N_{t}$. At each time slice the many-body propagator is linearized via the Hubbard-Stratonovich transformations [8]; observables are then calculated as expectation values in the canonical ensemble of nuclear states.

To circumvent the "sign problem" encountered in the SMMC calculations with realistic interactions, we use the extrapolation procedure outlined in [9]. One defines a set of Hamiltonians $H(g, \chi)=[1-(1-g) / \chi] H_{G}+g H_{B}$ such that $H(g=1, \chi)=H$ is the physical Hamiltonian and $H_{G, B}$ are the "good" and "bad" parts of the Hamiltonian, respectively. For $g \leq 0, H(g, \chi)$ is free of the sign problem; the matrix elements are therefore calculated for several values $g \leq 0$ and extrapolated to $g=1$. The value of $\chi$ is chosen to make the linear $g$ extrapolation as smooth as possible.

To validate our method, we calculated the matrix elements for ${ }^{48} \mathrm{Ca}$ in the complete $p f$ shell with the KB3 interaction [10] for six equally spaced $g$ values between -1.0 and 0.0 using $\chi=4$ and extrapolated to the physical result at $g=1$. Each calculation involved 2500-3500 Monte Carlo samples and was performed at $\beta=2 \mathrm{MeV}^{-1}$ with $N_{t}=48$. The direct diagonalization calculations for ${ }^{48} \mathrm{Ca}$ with which we compare our results were performed using an implementation of the Lanczos algorithm [11]. We calculated both the closure and exact matrix elements for the same Hamiltonians $H(g, \chi)$ as used in the SMMC.

We found the slope of $\ln [\phi(\tau, 0)]$ to be in good agreement with that expected from the difference of the energies for ${ }^{48} \mathrm{Ti}$ and ${ }^{48} \mathrm{Ca}$ (Fig. 1) and extracted $\left|M_{c}\right|$ from the intercept. The SMMC closure matrix elements for $g \leq 0$ are in very good agreement with the direct diagonalization results (Fig. 1) indicating that our temperatures are sufficiently low to correctly calculate the closure matrix element from the ground state of ${ }^{48} \mathrm{Ca}$ to ${ }^{48} \mathrm{Ti}$. However, the direct diagonalization calculations show a small curvature near $g=1.0$ that the extrapolation cannot reproduce. Our linear extrapolation of the closure matrix element, which
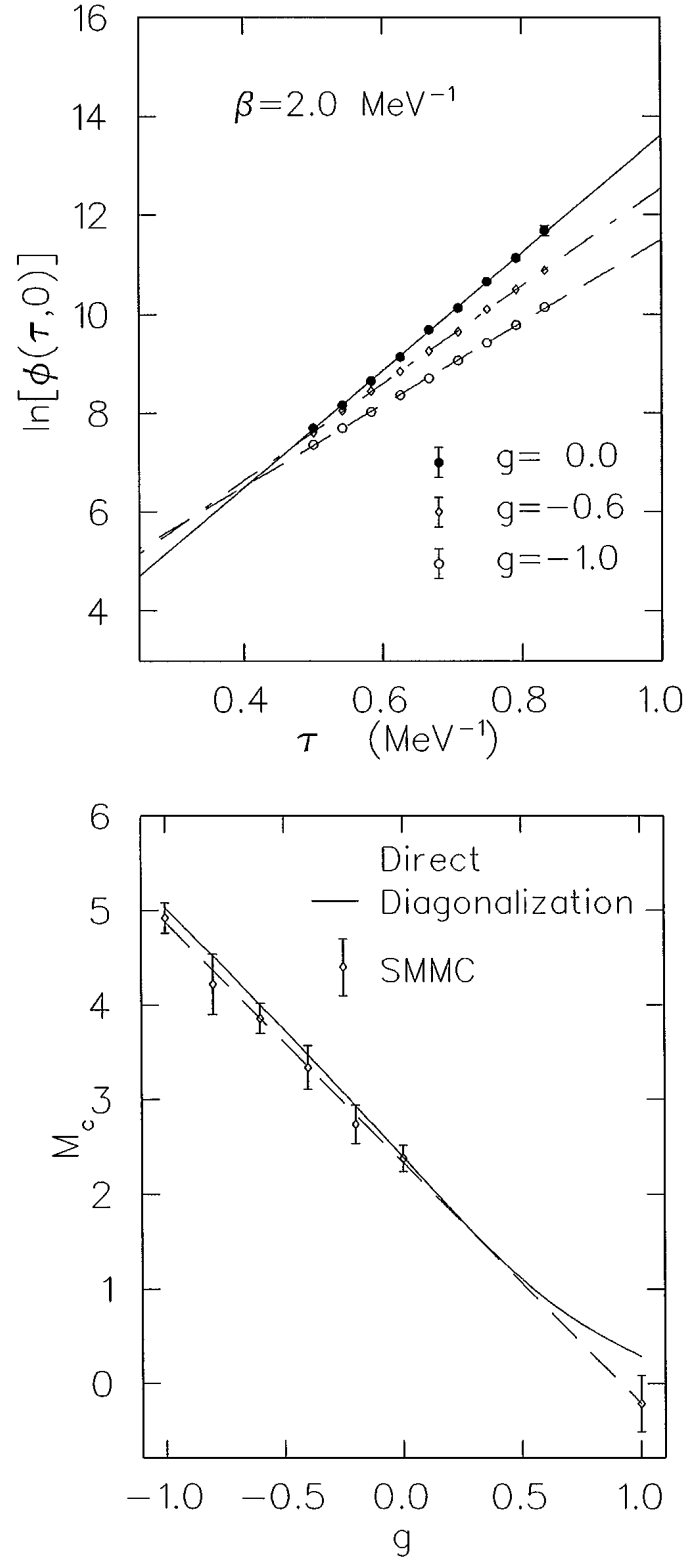

FIG. 1. Upper: $\ln [\phi(\tau, 0)]$ for ${ }^{48} \mathrm{Ca}$ calculated at $\beta=2.0 \mathrm{MeV}^{-1}$ with $N_{t}=48$. The lines are best fits. Lower: SMMC and direct diagonalization closure matrix elements for ${ }^{48} \mathrm{Ca}$. The SMMC points are linearly extrapolated to $g=1.0$.

takes place over almost a factor of 20 , therefore underestimates the physical $(g=1.0)$ calculation. We obtain $-0.21 \pm 0.29$ for the closure matrix element to be compared with the direct diagonalization result of 0.29 . As the natural scale for $M_{c}$ is given by the sum rule [2] as $\approx 21$, we may conclude that the SMMC successfully reproduces the shell model suppression of a factor of 70 .

The calculation of the function $\phi\left(\tau, \tau^{\prime}\right)$ was performed for $\tau=0.5 \mathrm{MeV}^{-1}$ and for thirteen $\tau^{\prime}$ values spaced equally between 0.0 and $0.5 \mathrm{MeV}^{-1}$. This combination of parameters was checked to give converged results 
for the matrix element. We then calculated $\eta(T, \tau)$ for thirteen values of $T \leq 0.5 \mathrm{MeV}^{-1}$; the upper limit of $T$ is sufficiently large for the integral in Eq. (3) to converge. From these, we obtained $M^{2 \nu}(T, \tau)$ [Eq. 6] as shown in Fig. 2 for some representative values of $g$ [12]. In Fig. 2, we show the good agreement between the SMMC matrix elements and direct diagonalization for $g \leq 0$. Even though the value of $\chi=4.0$ was chosen to make the linear extrapolation as smooth as possible, the direct diagonalization results still have a small curvature. For the exact $2 \nu$ matrix element we obtain an extrapolated
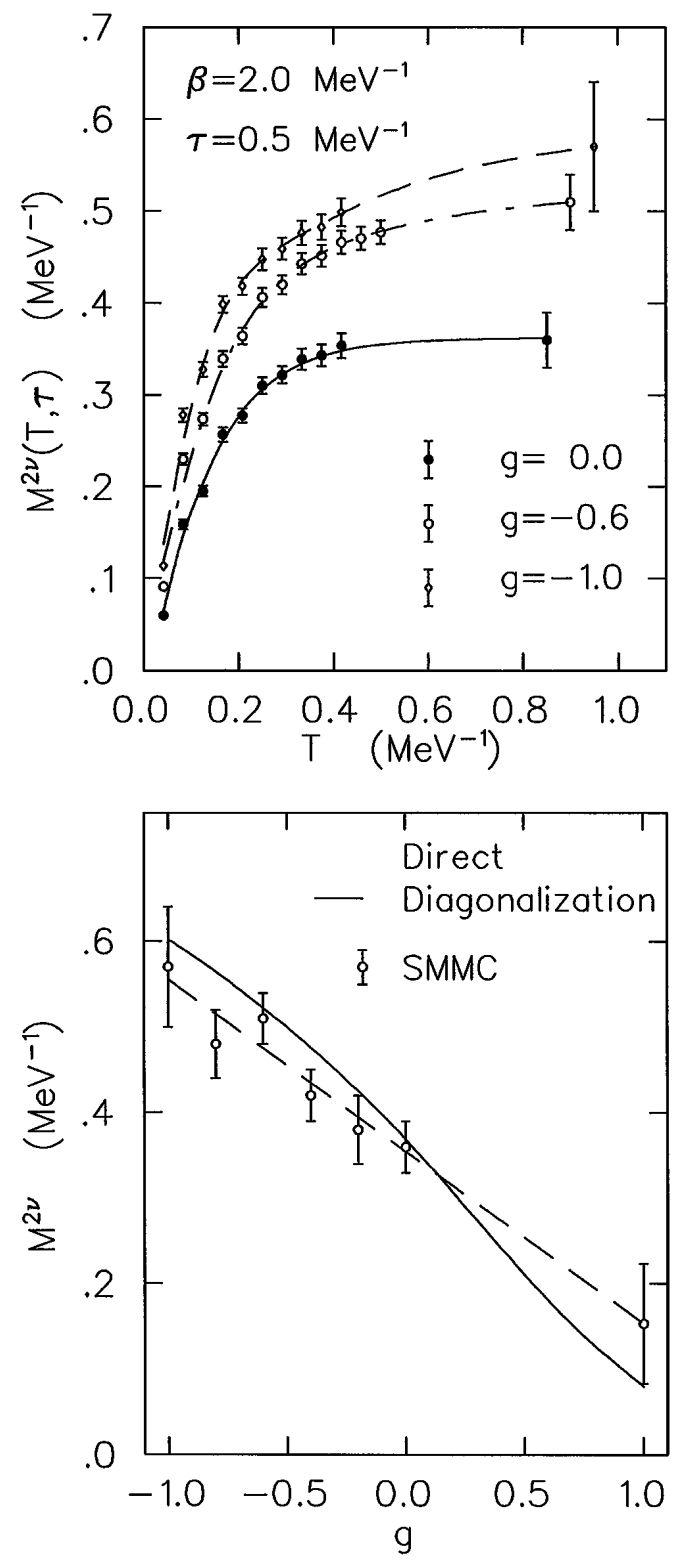

FIG. 2. Upper: $M^{2 \nu}(T, \tau)$ for ${ }^{48} \mathrm{Ca}$ calculated at $\beta=$ $2.0 \mathrm{MeV}^{-1}$ with $N_{t}=48$. The points at large $T$ show the asymptotic value $(T \rightarrow \infty)$ of the matrix elements obtained according to [12]; lines are drawn to guide the eye. Lower: SMMC exact matrix elements and the direct diagonalization results for ${ }^{48} \mathrm{Ca}$. The SMMC matrix elements are linearly extrapolated to $g=1.0$. value of $0.15 \pm 0.07 \mathrm{MeV}^{-1}$ whereas the calculation of Caurier, Poves, and Zuker [13] (including the erratum in [10]) gives $0.08 \mathrm{MeV}^{-1}$. There is thus agreement within the uncertainty.

We now apply the SMMC method to a heavier nucleus, where direct diagonalization is not possible. In particular, we calculate the $2 \nu$ matrix element for ${ }^{76} \mathrm{Ge}$ using an effective interaction based on the Paris potential in the $\left(0 f_{5 / 2}, 1 p, 0 g_{9 / 2}\right)$ orbitals, with the single particle energies taken from the levels of ${ }^{57} \mathrm{Ni}$ relative to the ${ }^{56} \mathrm{Ni}$ core [14]. This interaction has been constructed using a $G$ matrix folded-diagram method, in close analogy with the calculations carried out by Shurpin, Kuo, and Strottman [15] and by Dean et al. [4]. The model space comprises some $10^{8}$ configurations, so that our SMMC calculation is significantly larger than previous shell model treatments of ${ }^{76} \mathrm{Ge}$ [1]. While it avoids spurious excitations of the center of mass, it does not include all spin-orbit pairs of orbitals and thus does not obey the Ikeda sum rule for GT strengths. However, this model space (with the choice of an appropriate effective interaction) should adequately describe those low-lying states expected to be the most important for $2 \nu \beta \beta$ decay [16].

We performed the ${ }^{76} \mathrm{Ge}$ calculation at $\beta=2.5 \mathrm{MeV}^{-1}$ with $N_{t}=60$. The effective interaction used reproduces the experimental mass splitting of ${ }^{76} \mathrm{Ge}$ and ${ }^{76} \mathrm{Se}$ well: $21.35 \pm 0.30 \mathrm{MeV}$ compared to the experimental splitting of $20.72 \mathrm{MeV}$ (the Coulomb energy was calculated following Ref. [17]). The mass splitting of ${ }^{76} \mathrm{Ge}$ and other $A=$ 76 nuclei $\left({ }^{76} \mathrm{Zn},{ }^{76} \mathrm{Kr},{ }^{76} \mathrm{Sr}\right)$ compares favorably with the Coulomb corrected experimental values or value from systematics in the case of ${ }^{76} \mathrm{Sr}$. Our value for the $\beta^{-}$strength of ${ }^{76} \mathrm{Ge}$ is $B\left(\mathrm{GT}_{-}\right)=\left\langle\mathbf{G}^{\dagger} \cdot \mathbf{G}\right\rangle=19.09 \pm 0.39$, and we find an energy centroid of $6.3 \pm 0.2 \mathrm{MeV}$, while the experimental values are 19.9 and $9.1 \mathrm{MeV}$, respectively [18]; the apparent near consistency of this total strength with experiment is misleading as we have not employed the renormalization of $g_{A}=1.0$ and we have missing strength in our model space corresponding mainly to the transitions between the $g_{9 / 2}$ and $g_{7 / 2}$ orbitals. This missing high-energy strength should not affect the low-lying states of ${ }^{76} \mathrm{As}$ that are important for $2 \nu \beta \beta$ decay. Our value for the $\beta^{+}$strength for ${ }^{76} \mathrm{Se}$ is $B\left(\mathrm{GT}_{+}\right)=0.60 \pm 0.13$. This strength is identically zero in the independent particle model, and it is generated only by the smearing of the Fermi surface due to the interaction.

We performed two independent sets of calculations for both the closure and the exact matrix element using the $\chi=4$ and $\chi=\infty$ families of $H(g, \chi)$. The best-fit extrapolations to $g=1.0$ were linear for both the closure and the exact matrix elements in both cases. Our results for the closure matrix elements are $-0.36 \pm 0.34$ and $0.08 \pm$ 0.17 for $\chi=4$ and $\chi=\infty$, respectively. These are to be compared with the truncated shell model calculation of Haxton and Stephenson [1] (using a different effective interaction) that resulted in a value of 2.56 . 
We find consistent $M^{2 \nu}$ values for the $\chi=4$ and $\chi=$ $\infty$ cases (Fig. 3). Our results are $0.12 \pm 0.07$ and $0.12 \pm$ $0.06 \mathrm{MeV}^{-1}$, respectively (a combined value of $0.12 \pm$ $0.05 \mathrm{MeV}^{-1}$ ), while the experimental value of this matrix element (using $g_{A}=1.26$ ) is $0.14 \pm 0.01 \mathrm{MeV}^{-1}$ [5]. However, shell model calculations of ordinary $\beta$ decay consistently suggest that $g_{A}$ is renormalized to 1.0 in the nuclear medium [4], in which case the experimental matrix element is $0.22 \pm 0.01 \mathrm{MeV}^{-1}$.

There has been no previous shell model calculation of $M^{2 \nu}$. Haxton and Stephenson [1] obtained an estimate in the closure approximation by taking the average energy denominator to be the position of the $\beta^{-}$GT resonance in ${ }^{76} \mathrm{Ge}(9.4 \mathrm{MeV})$. We find significantly smaller values of $\bar{E} \equiv M_{c} / M^{2 \nu}(-3.0 \pm 3.3$ and $0.57 \pm 1.26 \mathrm{MeV}$ for $\chi=4$ and $\infty$, respectively), in agreement with other $2 \nu \beta \beta$ decay candidates such as ${ }^{48} \mathrm{Ca},{ }^{100} \mathrm{Mo}$, and ${ }^{128} \mathrm{Te}$ where $\bar{E}$ is known to be significantly smaller than the position of the $\beta^{-}$GT resonance [16].

In this Letter, we have demonstrated an SMMC method to calculate $2 \nu \beta \beta$ decay matrix elements and test it for the ${ }^{48} \mathrm{Ca}$ decay against direct diagonalization. We have also calculated the matrix element for ${ }^{76} \mathrm{Ge}$ in a model space significantly larger than previous calculations, and obtain a result that is in reasonable agreement with experiment. Although our extrapolations of the matrix elements may introduce systematic errors in our physical value, the fact that two independent calculations give consistent values enhances the confidence in our result. The dependence of $M^{2 \nu}$ on the effective interaction and single-particle energies remains to be investigated. A

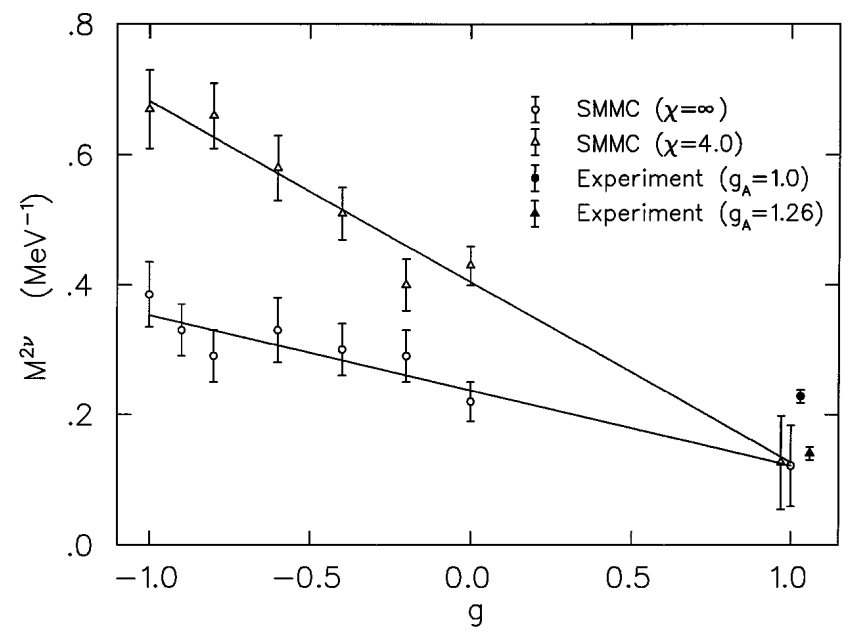

FIG. 3. SMMC exact matrix elements for ${ }^{76} \mathrm{Ge}$ calculated using the Hamiltonians $H(g, \chi)$ with $\chi=4$ and $\chi=\infty$. The lines are linear fits to the points in both cases. The extrapolated values and the experimental result of Ref. [5] are shown staggered around $g=1.0$ for clarity. more detailed description of these calculations will be given elsewhere, and work is in progress to calculate the matrix elements for several other, heavier nuclei.

This work was carried out under grants from the NSF and the DOE. Computational cycles were provided by the Concurrent Supercomputing Consortium on the Intel Touchstone Delta and the Intel Paragon and on the IBM SP2 at the Maui High Performance Computing Center.

[1] W. C. Haxton and G. J. Stephenson, Jr., Prog. Part. Nucl. Phys. 12, 409 (1984).

[2] M. Moe and P. Vogel, Ann. Rev. Nucl. Part. Sci. 44, 247 (1994).

[3] P. Vogel and M. R. Zirnbauer, Phys. Rev. Lett. 57, 3148 (1986).

[4] B. A. Brown and B. H. Wildenthal, At. Data Nucl. Data Tables 33, 347 (1985); K. Langanke, D. J. Dean, P.B. Radha, Y. Alhassid, and S.E. Koonin, Phys. Rev. C 52, 718 (1995); D. J. Dean, S. E. Koonin, T. T. S. Kuo, K. Langanke, and P.B. Radha, Phys. Lett. B 367, 17 (1996).

[5] A. Balysh et al., Phys. Lett. B 322, 176 (1994).

[6] F. Boehm and P. Vogel, Physics of Massive Neutrinos (Cambridge University Press, Cambridge, England, 1992), 2nd ed.

[7] G. H. Lang, C.W. Johnson, S.E. Koonin, and W.E. Ormand, Phys. Rev. C 48, 1518 (1993).

[8] J. Hubbard, Phys. Rev. Lett. 3, 77 (1959); R. L. Stratonovich, Dokl Akad. Nauk. SSSR [Sov. Phys. Dokl.] 115, 1097 (1957).

[9] D. J. Dean, S. E. Koonin, K. Langanke, P. B. Radha, and Y. Alhassid, Phys. Rev. Lett. 74, 2909 (1995).

[10] E. Caurier, A.P. Zuker, A. Poves, and G. MartinezPinedo, Phys. Rev. C 50, 225 (1994).

[11] E. Caurier, code ANTOINE, Strasbourg (1989).

[12] To determine the asymptotic $(T \rightarrow \infty)$ value of $M^{2 \nu}(\tau, T)$, we construct an ensemble of Gaussian-distributed $\phi\left(\tau, \tau^{\prime}\right)$ with mean and standard deviations as calculated in SMMC. Each $M^{2 \nu}(\tau, T)$ is then fit by the form $a-$ $b e^{-c T}$ between $T=0.17$ and $0.5 \mathrm{MeV}^{-1}$, and the average and standard deviation of $a$ gives the required matrix element and its error (shown at large $T$ in the upper panel of Fig. 2).

[13] E. Caurier, A. Poves, and A. P. Zuker, Phys. Lett. B 252, 13 (1990).

[14] A. Bohr and B. Mottelson, Nuclear Structure (Benjamin, New York, 1969), Vol. 1.

[15] J. Shurpin, T. T. S. Kuo, and D. Strottman, Nucl. Phys. A408, 310 (1983).

[16] M. Ericson, T. Ericson, and P. Vogel, Phys. Lett. B 328, 259 (1994).

[17] W.D. Myers and W. J. Swiatecki, Nucl. Phys. 81, 1 (1966).

[18] R. Madey et al., Phys. Rev. C 40, 540 (1989). 